Schmerz 2021 · 35:157-158

https://doi.org/10.1007/s00482-021-00553-6

Angenommen: 8. April 2021

(c) Springer Medizin Verlag GmbH, ein Teil von Springer Nature 2021

\author{
Stefanie Burghaus ${ }^{1}$. Winfried Häuser ${ }^{2,3}$ \\ 'Frauenklinik, Universitätsklinikum Erlangen, Erlangen, Deutschland \\ ${ }^{2}$ Medizinisches Versorgungszentrum für Schmerzmedizin und seelische Gesundheit Saarbrücken \\ St. Johann, Saarbrücken, Deutschland \\ ${ }^{3}$ Innere Medizin 1, Klinikum Saarbrücken gGmbH, Saarbrücken, Deutschland
}

\title{
Schmerztherapie - ein Stiefkind der Frauenheilkunde? Endometriose - ein Stiefkind der Schmerzmedizin?
}

Seit dem Editorial „Viszerale Schmerzen - ein Stiefkind der Schmerzmedizin“" [1] scheint sich auf den ersten Blick in der Schmerzmedizin in Bezug auf Unterbauchschmerzen im Allgemeinen und zur Endometriose im Besonderen wenig geändert zu haben. Beiträge zu chronischen Unterbauchschmerzsyndromen bei Frauen und Männern finden sich in Der Schmerz sehr selten [2]. Der letzte Beitrag zur Endometriose in dieser Zeitschrift ist aus dem Jahr 2016 [3]. Auf der Homepage der International Association for the Study of Pain (IASP) gibt es ein Fact Sheet zur Endometriose in verschiedenen Sprachen (jedoch nicht auf Deutsch) aus dem Jahr 2007 [4]. Das vom Arbeitskreis „Viszerale Schmerzen" der Deutschen Schmerzgesellschaft organisierte Symposium zu "Endometriose und Schmerz" ist den Kürzungen des Onlinekongresses 2020 zum Opfer gefallen. Im Programm finden sich bei den schmerzsyndrombezogenen Symposien „die üblichen Verdächtigen“ (Kopfschmerzen, muskuloskeletale Schmerzen, neuropathische Schmerzen).

In der Frauenheilkunde gibt es ein Defizit an epidemiologischen Studien zu chronischen Unterbauchschmerzen. Die einzige bisher in Deutschland durchgeführte Prävalenzstudie zum chronischen Unterbauchschmerz der Frau ermittelte eine altersassoziierte Häufigkeit von $12 \%$ mit häufigerem Auftreten bei jüngeren Patientinnen [5]. Zur Prävalenz und Inzidenz der Endometriose liegen aus Deutschland keine verlässlichen Zahlen vor. Daten zur Behandlungsprävalenz (d.h. dokumentierte Kontakte in einer Versorgungs- oder Behandlungseinrichtung) reichen von 0,8 bis $2 \%$ [6]. Die Diagnose einer Endometriose wird oft erst nach mehreren Jahren gestellt. Ärztliche Fehldiagnosen und Bagatellisierung der Schmerzen im sozialen Umfeld tragen zur Verzögerung der Diagnose bei [7]. Manche Patientinnen fühlen sich von ihren Bezugspersonen und Ärzt ${ }^{*}$ innen nicht ernst genommen [8]. In den S2-Leitlinien zum chronischen Unterbauschmerz [9] und zur Endometriose [6] war ein Mangel an hochwertigen Studien zur symptomatischen Schmerztherapie (Medikamente, Physiotherapie, Psychotherapie) im Vergleich zu hormonellen und operativen Therapien auffällig.

Doch es gibt auch Lichtblicke. An den beiden genannten Leitlinien der Frauenheilkunde wurde die Deutsche Schmerzgesellschaft beteiligt. Beide Leitlinien enthalten ein Kapitel zur Schmerztherapie [6,9]. An der zweiten Aktualisierung der S3-Leitlinie „Langzeitanwendung von Opioiden bei chronischen nichttumorbedingten Schmerzen" (LONTS) hat sich auch die Deutsche Gesellschaft für Gynäkologie und Geburtshilfe beteiligt $[10,11]$. Im Kapitel „Potenzielle Indikationen" wurden neu chronische Unterbauchschmerzen bei multilokulärer Endometriose aufgenommen [11]. Der Schriftleiter der Zeitschrift Der
Schmerz hat einem Themenheft zur Endometrioseleitlinie zugestimmt, das Sie gerade in Händen halten bzw. auf Ihrem Monitor lesen. Sie finden eine Manuskriptfassung der drei Vorträge, die auf dem Deutschen Schmerzkongress 2020 nicht gehalten werden konnten.

Frau Burghaus, Sprecherin der Steuergruppe der Endometrioseleitlinie, wird einen Überblick darüber geben, was Nichtfrauenärzt ${ }^{*}$ innen über Endometriose wissen sollten [12].

Frau Mechsner wird einen Überblick über die Empfehlungen des Kapitels "Schmerztherapie“ der Leitlinie geben. Sie wird die aktuelle Studienlage zur symptomatischen Schmerztherapie der Endometriose zusammenfassen und auch ihre eigenen umfangreichen klinischen Erfahrungen im Endometriosezentrum der Charité - Universitätsmedizin Berlin darstellen [13].

Herr Häuser wird die mögliche Überlappung von chronischem Unterbauchschmerz bei Endometriose mit anderen chronischen Schmerzsyndromen wie Spannungskopfschmerzen und Fibromyalgiesyndrom und die möglichen Konsequenzen im Falle von chronischen überlappenden Schmerzsyndromen für die Behandlung der betroffenen Frauen darstellen [14].

Die Autor*innen wünschen sich, dass die Beiträge sowohl den Blickwinkel der Leser*innen in ihrem Fachgebiet als auch die Zusammenarbeit von Frauenärzt*innen mit Schmerz- 
mediziner*innen fördern und $\mathrm{zu}$ einer besseren interdisziplinären Versorgung von Patientinnen mit chronischen Unterbauchschmerzen und Endometriose beitragen.

\section{Stefanie Burghaus}

S. Burghaus

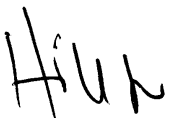

\section{W. Häuser}

\section{Korrespondenzadresse}

\section{Prof. Dr. Winfried Häuser}

Innere Medizin 1, Klinikum Saarbrücken $\mathrm{gGmbH}$

Winterberg 1, 66119 Saarbrücken, Deutschland whaeuser@klinikum-saarbruecken.de

Interessenkonflikt. S. Burghaus und W. Häuser geben an, dass kein Interessenkonflikt besteht.

\section{Literatur}

1. Jänig W, Häuser W (2014) Viszerale Schmerzen. Immer noch ein Stiefkind der Schmerzmedizin? Schmerz 28:230-232

2. Siedentopf F, Sillem M (2014) Chronischer Unterbauchschmerz der Frau. Schmerz 28:300-304

3. Mechsner S (2016) Endometriose: Eine oft verkannte Schmerzerkrankung. Schmerz 30:477-490

4. International Association for the Study of Pain Endometriosis and its association with other painful conditions. https://www.iasp-pain.org/ Advocacy/Content.aspx?ItemNumber $=1107$. Zugegriffen:5. Dez. 2020

5. Beutel ME, Weidner K, Brähler E (2005) Chronic pelvic pain of Women and its Co-Morbidity. Geburtshilfe Frauenheilkd 65:61-67

6. Deutsche Gesellschaft für Gynäkologie und Geburtshilfe (2020) Diagnostik und Therapie der Endometriose. https://www.awmf.org/leitlinien/ detail/Il/015-045.html.Zugegriffen:4.Dez. 2020

7. Hudelist G, Fritzer N, Thomas A, Niehues C, Oppelt $P_{\text {, }}$ Haas D, Tammaa A, Salzer H (2012) Diagnostic delay for endometriosis in Austria and Germany: causes and possible consequences. Hum Reprod 27:3412-3416

8. Kriesl I (2018) Zwischen Schmerz und Hoffnung "Ärzte haben mich nicht ernst genommen": Fünf Frauen erzählen über ihr Leben mit Endometriose. https://www.stern.de/gesundheit/leben-mitendometriose---fuenf-frauen-erzaehlen-vonihrer-erkrankung-8181284.html. Zugegriffen: 9. Dez. 2020

9. Deutsche Gesellschaft für Psychosomatische Frauenheilkunde und Geburtshilfe (2021) Chronischer Unterbauchschmerz der Frau. https://www.awmf. org/leitlinien/detail/II/016-001.html.Zugegriffen: 4. Dez. 2020
10. Häuser W, Klose P, Welsch P, Petzke F, Nothacker M (2020) Leitlinienreport der zweiten Aktualisierung der S3-Leitlinie "Langzeitanwendung von Opioiden bei nicht-tumorbedingten Schmerzen LONTS". Schmerz 34:245-278

11. Häuser W, Bock F, Hüppe $M$, Nothacker $M$, Norda $H$, Radbruch L, Schiltenwolf M, Schuler M, Tölle T, Viniol A, Petzke F; Koautoren für die Konsensusgruppe der 2. Aktualisierung derS3-Leitlinie LONTS (2020) Empfehlungen der zweiten Aktualisierung der Leitlinie LONTS: Langzeitanwendung von Opioiden bei chronischen nicht-tumorbedingten Schmerzen. Schmerz 34:204-244

12. Burghaus S, Beckmann MW (2021) Endometriose - gynäkologische Diagnostik und Therapie. Schmerz. https://doi.org/10.1007/s00482-02100541-w

13. Mechsner S (2021) Endometrioseschmerz beherrschen. Schmerz. https://doi.org/10.1007/s00482021-00543-8

14. Häuser W (2021) Endometriose und chronische überlappende Schmerzsyndrome. Schmerz. https://doi.org/10.1007/s00482-021-00535-8
Franziska-Tiburtius-Preis: Ärztinnen auf neuen Wegen

Ausschreibung

Die Women's Networking Lounge e.V. (WNL) vergibt erstmalig den FranziskaTiburtius-Preis. In Referenz an die Namensgeberin des Preises werden Medizinerinnen gesucht, die neue Wege gehen - Pionierinnen, die etwas wagen. Gefragt sind Präventionskonzepte, Kooperationsmodelle, E-Medizin, soziale Projekte in der Medizin, neue Formen der Patientenkommunikation oder andere innovative Ideen. Teilnehmen können Ärztinnen und Zahnärztinnen aus Deutschland. Der Award ist mit $10.000 €$ dotiert.

Christine Wernze, Vorstandsvorsitzende der WNL zum Award: „Mit der Vergabe dieses Preises geht es uns darum, Ärztinnen zu zeigen, die neben ihrem alltäglich grossartigen Dienst in unserem Gesundheitssystem die Kraft aufbringen, eine besondere Idee zu verfolgen, die das System insgesamt nachhaltig prägt und noch besser macht."

Franziska Tiburtius war die erste Ärztin in Deutschland, als sie sich 1877 in Berlin niederließ. Zuerst führte sie zusammen mit der Zahnärztin Henriette PagelsenHirschfeld eine Gemeinschaftspraxis. Danach eröffnete Franziska Tiburtius mit ihrer Studienkollegin Emilie Lehmus in Prenzlauer Berg eine Poliklinik für Frauen und Kinder. Beide hatten in Zürich studiert, da Frauen bis zu einem entsprechenden Bundesratsbeschluss im Jahr 1899 der Zugang zu deutschen Universitäten verwehrt war.

Bewerbungen sind bis zum 31.08.2021 über die WNL-Website möglich

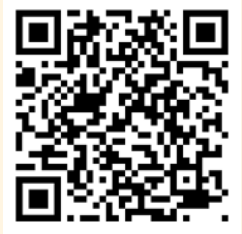

www.womensnetworkinglounge.de 